\title{
Falla hepática fulminante por virus Epstein Barr. Rol de los corticoides. Caso clínico
}

\author{
PAULA VIVIANI G. ${ }^{1}$, CAMILA SALAS A. ${ }^{1}$, JORGE ROQUE E. ${ }^{2}$ \\ 1. Becada de Pediatría, Clínica Alemana de Santiago, Facultad de Medicina Universidad del Desarrollo. Santiago de Chile. \\ 2. Unidad Cuidados Intensivos de Pediatría, Clínica Alemana de Santiago.
}

\begin{abstract}
Epstein Barr virus and acute liver failure. Role of steroids. Clinical case

Acute liver failure (ALF) due to Epstein Barr Virus (EBV) is rare in immunocompetent patients. The role of steroids in this case is not well defined and remains controversial. Case report: 7 years old female presenting with unspecific respiratory symptoms for 2 weeks, fever, lymphadenopathy, jaundice and dark brown urine. Total bilirubin: 9 and direct: 6.3, alkaline phosphatases: 523; AST: 7.527, ALT: 6.537; Prothrombin (PT): 17\%, INR: 4.7; ammonium 510 and glucose 33. Abdominal ultrasound: normal liver and splenomegaly. Monotest Positive. She was transferred to a liver transplant centre (LT). Lab results at admission: PT $21 \%$, bilirubin 9.8, AST 2717, ALT 3.716 and ammonium 177. EEG with diffuse and slowing conductivity consistent with encephalopathy. Positive IgM EBV, other aetiologies were ruled out. She was activated for LT due to ALF and while in waiting list methylprednisolone was administered for 5 days. She evolved with normalization of liver tests and clinical improvement. Conclusion: In this case the use of steroids was associated with a rapid and favourable clinical and laboratory response without negative side effects. As in other presentations of serious infection by EBV, should consider the use of steroids in ALF due to EBV.
\end{abstract}

(Key words: Acute liver failure, Epstein Barr virus, steroids).

Rev Chil Pediatr 2013; 84 (3): 313-317

\section{RESUMEN}

La falla hepática fulminante (FHF) debida a Virus Epstein Barr (VEB) es poco frecuente en inmunocompetentes. La utilidad de los esteroides en este cuadro no ha sido definida y permanece muy controversial. Objetivo: Reportar el caso de una paciente con mononucleosis infecciosa por VEB que presenta FHF y es tratada con corticoides. Caso clínico: Escolar con cuadro de 2 sem de síntomas respiratorios altos, fiebre, adenopatías, con ictericia y orina oscura. Bilirrubina total: 9; B. Directa: 6,3; Fosfatasas Alcalinas: 523; GOT: 7.527; GPT: 6.537; Protrombina (PT): 17\%; INR: 4,7; Amonio 510 y glicemia 33. Ecografía abdominal hígado normal y esplenomegalia. Monotest Positivo. Se transfirió a centro de trasplante hepático (TH). Laboratorio de ingreso

Recibido el 10 de agosto de 2012, devuelto para corregir el 20 de febrero de 2013, segunda versión 20 de marzo de 2013 , aceptado para publicación el 08 de abril de 2013.

Este trabajo cumple con los requisitos sobre consentimiento /asentimiento informado, comité de ética, financiamiento, estudios animales y sobre la ausencia de conflictos de intereses según corresponda. 
PT 21\%; bilirrubina en 9,8; GOT 2717; GPT 3716 y amonio 177. EEG con enlentecimiento difuso compatible con encefalopatía grado 1. IgM VEB positiva, descartándose otras etiologías. Se activó para TH por FHF y mientras se administró Metilprednisolona por 5 días. Evolucionó con normalización de las pruebas hepáticas y mejoría clínica. Conclusión: En este caso el uso de esteroides se asoció a una rápida y favorable respuesta tanto clínica como de laboratorio sin presentar efectos secundarios negativos. Al igual que en otras presentaciones de infección grave por VEB, debiera considerarse el uso de esteroides en FHF por VEB.

(Palabras clave: Fallo hepático fulminante, virus Epstein Barr, Corticoides).

Rev Chil Pediatr 2013; 84 (3): 313-317

\section{Introducción}

La falla hepática fulminante (FHF) es una entidad poco frecuente en pediatría pero de extrema gravedad. Se define como la evidencia bioquímica de daño hepático, sin historia de enfermedad hepática conocida, coagulopatía que no se corrige con vitamina K con INR mayor a 1,5 si el paciente presenta encefalopatía o INR mayor a 2,0 en ausencia de ésta ${ }^{1}$. Tiene múltiples causas (infecciones, auto inmunidad, enfermedades metabólicas, drogas y toxicidad), quedando alrededor de un $50 \%$ de los pacientes sin un diagnóstico etiológico conocido $^{1-3}$. Su mortalidad alcanzaba hasta un $80 \%$ en la era previa al transplante hepático $(\mathrm{TH})$, con una sobrevida que mejoró hasta $70-90 \%$ con la introducción de esta nueva técnica ${ }^{2}$.

Dentro de los cuadros de FHF secundarios a infecciones virales, la infección por Virus Epstein Barr (VEB) se encuentra como una causa infrecuente, entre un $2 \%$ a $17 \%$, presentándose en la mayoría de los casos en el contexto de pacientes inmunodeprimidos (transplantados o con inmunodeficiencias congénitas o adquiridas) $)^{5}$. El VEB es un virus DNA de la familia Herpesviridae, altamente prevalente, llegando hasta un $90 \%$ de seropositividad en la población adulta ${ }^{5,6}$. Es el principal agente causal de la mononucleosis infecciosa (MNI) y durante la evolución de esta patología se produce hasta en un $90 \%$ de los pacientes alteración transitoria de las bioquímica hepática, generalmente asintomática ${ }^{6}$. La FHF debido a infección por VEB fue reportada por primera vez el año 1963 en un caso clínico que resultó fatal ${ }^{7}$. Desde entonces han aparecido nuevos reportes de casos en quienes se ha usado corticoides y/o antivirales para tratar esta enfermedad tanto en pacientes inmunocompetentes como inmunocomprometidos, cuyos resultados hasta ahora han sido dispares y controversiales ${ }^{5,8}$.

Nuestro objetivo es reportar el caso de una paciente con MNI por VEB que desarrolló una FHF, en quién se usó esteroides como tratamiento y revisar el estado actual de la literatura sobre este tema.

\section{Caso clínico}

Escolar de 7 años, sexo femenino, con antecedentes de asma y rinitis alérgica. Presentó cuadro de 2 sem de evolución de síntomas respiratorios altos, en el que se describe una adenopatía cervical izquierda sensible asociada a fiebre, motivo por el cual alcanzó a recibir amoxicilina-ácido clavulánico por 3 días. Desarrolló compromiso del estado general, somnolencia, vómitos, rechazo alimentario, palidez, ictericia y orina oscura, por lo cual se hospitalizó y solicitó exámenes de laboratorio: proteína $\mathrm{C}$ reactiva: $30 \mathrm{mg} / \mathrm{L}$; VHS: 23 ; hematocrito $34 \%$; plaquetas 153.000 ; leucocitos 14.800 con linfocitos: 56\% y PMN: $31 \%$, e hipoprotrombinemia grave. Función renal, electrolitos plasmáticos y gases arteriales normales (tabla 1 día 1). Ecografía cervical demostró múltiples adenopatías bilaterales no abscedadas; ecografía abdominal sólo con esplenomegalia. Estudio para VEB con Monotest positivo. Dado exámenes de laboratorio y clínica compatibles con FHF, se derivó a un centro de trasplante hepático, donde se manejó inicialmente con hidratación parenteral, régimen hipoprotéico, n-acetil cisteína, lactulosa, norfloxacino, protección gástrica con omeprazol y vitamina K. Exámenes de control 
Tabla 1. Exámenes de laboratorio

\begin{tabular}{|cccccccc|}
\hline & GOT & GPT & Bili T & PT & INR & LDH & Amonio \\
\hline Día 1 & 7.527 & 6.537 & 9,09 & 17 & 4,7 & & 510 \\
\hline Día 2 & 2.870 & 3.942 & 8,95 & 21 & 3,2 & 1.877 & 177 \\
\hline Día 3 & 1.558 & 2.819 & 9,86 & 20 & 3,4 & 1.166 & 136 \\
\hline Día 4 & 517 & 1.924 & 6,56 & 28 & 2,5 & 896 & 116 \\
\hline Día 5 & 303 & 1.581 & 5,79 & 38 & 1,8 & 681 & 105 \\
\hline Día 6 & 134 & 1.151 & 3,95 & 75 & 1,2 & 646 & 497 \\
\hline Día 7 & 70 & 809 & 2,71 & 91 & 1 & $4: 5$ \\
\hline
\end{tabular}

GOT: Aspartato amino transferasa (U/L); GPT: Glutamato piruvato transaminasa (U/L); BiliT: bilirrubina total (mg/dL); PT: protrombina (\%); INR: International Normalization Ratio; LDH: lactato deshidrogenasa (U/L)

en tabla 1, día 2. EEG con enlentecimiento difuso compatible con encefalopatía grado 1 . Se activó como candidata a TH dado encefalopatía asociada a INR 3,4 que no corrigió con vitamina $\mathrm{K}$. TAC de cerebro con difusión sin edema ni hemorragia. Se re-confirmó etiología por VEB con IgM (+) para el mismo. Fue evaluada por inmunólogo, hepatólogo e infectólogo. Marcadores de autoinmunidad negativos y ferritina normal. Se descartó Enfermedad de Wilson, Virus hepatitis A, B y C, Parvovirus B19, CMV y VIH, y se decidió administrar corticoides (tabla 2). Clínicamente la paciente evolucionó hacia la mejoría, así como también las pruebas hepáticas de control (tabla 1) completando 5 días de tratamiento esteroidal.

\section{Discusión}

El uso de amoxicilina raramente se ha relacionado con el desarrollo de lesión hepatocelular, que en general se describe de grado leve, sin embargo, este riesgo aumenta a 1,7 casos por 10.000 prescripciones en la combinación con ácido clavulánico ${ }^{9}$. Un análisis en 69 pacientes con hepatotoxicidad por amoxicilina-ácido clavulánico incluidos en el registro español de hepatotoxicidad encontró que la expresión bioquímica de la lesión por este agente está claramente influenciada por la edad, predominando el tipo hepatocelular en los más jóvenes y el colestásico/mixto en mayores de 55 años ${ }^{10}$. En todo caso la lesión que se describe con más frecuencia es la hepatitis aguda
Tabla 2. Tratamiento administrado de metilprednisolona

Día 1: $2 \mathrm{mg} / \mathrm{kg} /$ dosis cada $6 \mathrm{~h}$

Día 2: $1 \mathrm{mg} / \mathrm{kg} /$ dosis cada $6 \mathrm{~h}$

Días 3,4 y 5 : 0,6 mg/kg/ dosis

colestásica, supuestamente producida por un mecanismo de idiosincrasia inmunoalérgica. Otra característica importante es que su riesgo de daño aumentaría frente a exposiciones repetidas a este fármaco, y que la lesión puede aparecer hasta 6 sem después de suspendida la amoxicilina-ácido clavulánico, así como que el tiempo medio de resolución del cuadro es de $8 \mathrm{sem}$. No se ha descrito tratamiento específi$\mathrm{co}^{9}$. En este caso la paciente alcanzó a recibir sólo 3 días de tratamiento con amoxicilina-ácido clavulánico, sin antecedentes de uso previo y el cuadro de FHF apareció casi de inmediato, lo que no concuerda con el patrón descrito previamente de daño por esta droga, así como tampoco la gravedad y la rápida mejoría posterior.

El uso de corticoides en casos de infecciones por VEB se debiera reservar para situaciones específicas, tales como obstrucción incipiente de vía aérea, anemia hemolítica aguda, compromiso cardíaco grave o enfermedad neurológica ${ }^{11}$. siendo casi inexistente la evidencia respecto al uso de esteroides en casos de FHF por VEB. Sin embargo, se usa esteroides sistémicos en el FHF de origen autoinmune, con lo que rápidamente se logra una mejoría clínica y 
de laboratorio, evitando el TH en la gran mayoría de estos $\operatorname{casos}^{12}$. Por otro lado, el riesgo del uso de esteroides en el FHF es que puede favorecer la aparición de infecciones sistémicas oportunistas graves previo y /o posterior al potencial $\mathrm{TH}$, generando una mayor morbimortalidad $^{4}$.

Se describen 3 potenciales mecanismos de daño hepático asociados a la infección por VEB: hepatotoxicidad directa por el virus; expansión descontrolada monoclonal de linfocitos $\mathrm{T}$ asociada a disfunción de los linfocitos natural killer con liberación de citoquinas en respuesta a la infección inicial por VEB a los linfocitos B (mecanismo de autoinmunidad); y el síndrome hemofagocítico secundario que corresponde a una proliferación descontrolada de linfocitos e histiocitos con una producción exagerada de citoquinas ${ }^{7,8}$.

En el primer mecanismo no habría utilidad de la terapia con esteroides, aunque no hay evidencia concreta de este tipo de daño hepático. En el caso de la expansión monoclonal descontrolada de linfocitos $\mathrm{T}$ si se podría plantear la utilidad de los corticoides al igual que en el síndrome hemofagocítico, actuando como inmunomoduladores en estos 2 mecanismos donde la inmunidad esta desregulada. Además en el síndrome hemofagocítico, se ha reportado incluso el uso de inmunosupresores como la ciclosporina $\mathrm{A}^{13}$ o quimioterapia (Etopósido, Metotrexato o Vinblastina) con buena respuesta en los casos asociados a infección por $\mathrm{VEB}^{14}$.

Con respecto al uso de antivirales como el Ganciclovir, no existe evidencia clínica que soporte su uso en casos de FHF por VEB, sin embargo, hay reportes en la literatura de su utilización en casos de infecciones graves por VEB en pacientes inmunosuprimidos, como por ejemplo con compromiso infeccioso encefálico o también en FHF por VEB post transplante hepático ${ }^{15}$. Adams et al, reporta dos casos de hepatitis grave en pacientes adultos por VEB en los cuales se utilizó ganciclovir como tratamiento con buen resultado ${ }^{7}$, sin embargo, no encontramos reportes en pacientes pediátricos que señalen lo mismo.

Por otro lado, la resolución espontánea de FHF no asociado a ingesta de paracetamol es por lo general baja (menor al 20\% según algunas series) ${ }^{3}$ lo que en este caso sumado a la relación temporal de la mejoría clínica y de laboratorio asociada a la administración de metilprednisolona no deja de ser llamativo.

Queda la necesidad de evidencia clínica que demuestre cual es el efecto real del uso de corticoides en casos de FHF debido a infección por VEB, pero siendo esta una entidad tan infrecuente, sólo queda la experiencia de reportes de casos aislados ${ }^{8}$.

En resumen, presentamos el caso clínico de una paciente de 7 años, inmunocompetente que desarrolló una FHF por infección VEB en el contexto de una MNI, en quien se administró corticoides sistémicos lo cual se asoció a una rápida mejoría tanto clínica como de laboratorio, evitándose el transplante hepático. No hubo efectos secundarios negativos en esta paciente atribuibles al uso de corticoides.

\section{Referencias}

1.- Bucuvalas J, Yazigi N, Squires R: Acute Liver Failure in Children. Clin Liver Dis 2006; 10: 149-68.

2.- Roque J, Ríos G, Pinochet $C$, et al: Falla Hepática fulminante en Niños. Rev Chil Pediatr 2009; 80 (2): 143-8.

3.- Squires R, Schneider B, Bucuvalas J, et al: Acute Liver Failure in Children: the first 348 patients in the pediatric acute liver failure study group. J Pediatr 2006, 148 (5): 652-8.

4.- Hepp J, Zapata R, Buckel J, et al: Trasplante hepático en Chile: Aspectos generales, indicaciones y contraindicaciones (Documento de consenso). Rev Med Chile 2008; 136: 793-804.

5.- Aguilar J, Huaman M, Brar I: Ebstein Barr Virus Induced Fulminant Hepatitis in a Young Inmunocompetent Male: Case Report and Literature Review. Infect Dis Clin Prac 2009; 17: 295-7.

6.- Cisneros J, Herrero-Romero M: Hepatitis por virus del grupo Herpes. Enferm Infecc Microbio Clin 2006; 24 (6): 392-8.

7.- Allen $U$, Bass B: Fatal hepatic necrosis in glandular fever. J Clin Path 1963; 16: 337-41.

8.- Adams L, Deboer B, Jeffrey G, et al: Ganciclovir and the treatment of Epstein-Barr virus hepatitis. J Gastroenterol Hepatol 2006; 21: 1758-60.

9.- García-Cortés M, Andrade RJ, Lucena MI, et al: Hepatotoxicidad secundaria a fármacos de uso común. J 
Gastroenterol Hepatol 2005; 28 (8): 461-72.

10.- Robles MJ, Andrade RJ: Hepatotoxicidad por antibióticos: actualización en 2008. Rev Esp Quimioter 2008; 21 (4): 224-33.

11.- Cohen JI: Epstein-Barr Virus Infection. New England Journal of Medicine 2000; 343: 481-92.

12.- Prados J, Antonaya M, del Rey P, et al: Hepatitis Autoinmune: Análisis de una serie pediátrica. An Esp Pediatr 2001; 54 [Supl 3]: 17-20.

13.- Astigarriaga I, Fernández-Teijeiro A, García N, et al:
Síndrome hemofagocítico en la infancia: A propósito de 4 casos. An Esp Pediatr 1998; 49: 289-93.

14.- Verdugo L, Rodríguez Z, Tordecilla C, et al: Síndrome hemofagocítico secundario en pediatría. Experiencia clínica en ocho casos. Rev Chil Pediatr 2005; 76 (4); 397-403.

15.- Feranchak A, Tyson RW, Narkewicz MR, et al: Fulminant Epstein-Barr viral hepatitis: orthotopic liver transplantation and review of the literature. Liver Transplant Surg 1998: 4: 469-76. 